\title{
Overcoming 5-Fu Resistance of Colon Cells through Inhibition of Glut1 by the Specific Inhibitor WZB117
}

\author{
Wei Liu ${ }^{1 *}$, Yong Fang ${ }^{2}$, Xiao-Tong Wang ${ }^{1}$, Ju Liu' ${ }^{1}$ Xing Dan ${ }^{3}$, Lu-Lu Sun ${ }^{1}$
}

\begin{abstract}
Background: 5-Fluorouracil (5-FU) is the most commonly used drug in colon cancer therapy. However, despite impressive clinical responses initially, development of drug resistance to 5-Fu in human tumor cells is the primary cause of failure of chemotherapy. In this study, we established a 5-Fu-resistant human colon cancer cell line for comparative chemosensitivity studies. Materials and Methods: Real time PCR and Western blotting were used to determine gene expression levels. Cell viability was measured by MTT assay. Glucose uptake was assess using an Amplex Red Glucose/Glucose Oxidase assay kit. Results: We found that 5-Fu resistance was associated with the overexpression of Glut 1 in colon cancer cells. 5-Fu treatment at low toxic concentration induced Glut1 expression. At the same time, upregulation of Glut1 was detected in 5-Fu resistant cells when compared with their parental cells. Importantly, inhibition of Glut1 by a specific inhibitor, WZB117, significantly increased the sensitivity of 5-Fu resistant cells to the drug. Conclusions: This study provides novel information for the future development of targeted therapies for the treatment of chemo-resistant colon cancer patients. In particular it demonstrated that Glut1 inhibitors such as WZB117 may be considered an additional treatment options for patients with 5-Fu resistant colon cancers.
\end{abstract}

Keywords: 5-Fluorouracil - colon cancer - glut1 - specific inhibitor - WZB117

Asian Pac J Cancer Prev, 15 (17), 7037-7041

\section{Introduction}

5-fluorouracil (5-Fu) is a chemotherapeutic drug used worldwide in the treatment of metastatic colorectal cancer, either alone or in combination with other chemotherapeutic agents (Herrmann, 1996; Longley et al., 2003). Over the past 40 years, 5 -fluorouracil has been almost the sole chemotherapeutic option for patients with advanced colorectal cancer (DeBraud et al., 1998; Herrmann, 1996). The well-established mechanism for this anticancer agent is that treatment of cells with 5-Fu causes DNA damage. It inhibits thymidylate synthase as its main mechanism of action, leading to depletion of dTTP (Peters et al., 2000; Curtin et al., 1991). In addition, 5-FU has a complicated mechanism of action with several enzymes involved in its metabolic activation (Boyer et al., 2004; Zhao et al., 2014). Moreover, overexpression of thymidylate synthase has been shown to be associated with 5-FU resistance in colorectal cancer (Subbarayan et al., 2010). However, patients who respond to 5-FU ultimately become resistant (Zhang et al., 2008; Herrmann, 1996). Thus, resistance to 5 -FU has been a major obstacle in advanced colorectal cancer chemotherapy.

One hallmark of cancer cell is that unlike their normal counterparts, cancer cells use aerobic glycolysis with reduced mitochondrial oxidative phosphorylation for glucose metabolism. As a result, most cancers display upregulated glucose transport and aerobic glycolysis regardless of their oxygen status (Zhao et al., 2013; Li et al., 2014). In addition, cancer cells are found to be sensitive to glucose deprivation due to the dependence on glucose as major nutrition supply (Vander et al., 2009). The increased glucose transport in cancer cells has been attributed primarily to the upregulation of glucose transporter 1 (Glut1) that are responsible for basal glucose transport in almost all cell types (Carvalho et al., 2011). It has been reported that knockdown of Glut 1 by shRNA chemo-sensitized head and neck cancer cells to cisplatin both in vitro and in vivo (Wang et al., 2013), indicating inhibition of Glut1 might be the target for overcoming cancer chemoresistance. WZB117 is a novel Glut1 Inhibitor reported by a recent study which described that WZB117 is effective both in vitro and in vivo in inhibiting cancer cell growth and can serve as a novel compound for the further development of anticancer therapeutics (Liu et al., 2012).

In the present study, we sought to gain further insight into the mechanisms underlying 5-Fu resistance and cancer cell glucose metabolism. We established a 5-Furesistant human colon cancer cell line and used these cells in comparative chemosensitivity studies. We found that 5-FU resistance was associated with overexpression of

${ }^{1}$ Department of Pharmacy, Beijing Shijitan Hospital, Capital Medical University, ${ }^{2}$ Department of Pharmacy, Beijing Shijitan Hospital, Capital Medical University, Beijing, ${ }^{3}$ Northeast Pharmaceutical Group Sales Co., Ltd., Shenyang, China \& Equal contributors *For correspondence: Liuwei140327@163.com 
Wei Liu et al

Glut 1 in colon cancer cells. 5-Fu treatment at low toxic concentration induces Glut1 expression and inhibition of Glut 1 by specific inhibitor WZB117 resensitizes 5-Fu resistant cells to $5-\mathrm{Fu}$.

\section{Materials and Methods}

\section{Cell culture and antibodies}

CRC cell lines, HT-29 and SW48 were obtained from American Type Culture Collection (ATCC). Cell lines were maintained in Dulbecco's modified Eagle's medium (DMEM) with l-glutamine supplemented with $10 \%$ fetal bovine serum, $50 \mathrm{U} / \mathrm{ml}$ streptomycin-penicillin, $1 \%$ nonessential amino acid and HPEBS. Cells were incubated at $37^{\circ} \mathrm{C}$ with $5 \% \mathrm{CO} 2$ to $60-70 \%$ confluence.

Antibodies used from this project were purchased from: $\beta$-actin (Cell Signaling \#4967); GLUT1 (Santa Cruz: sc-7903). 5-Fu and WZB117 were purchased from Sigma-aldrich, Hong-kong, China.

\section{Generation of 5-Fu resistant cell lines}

The 5-Fu resistant cell lines are originally from HT-29 cells. Briefly, 5-Fu resistant cells were developed from parental HT-29 cells by treated with gradually increasing concentrations of 5-Fu in regular cell culture medium. The initial 5-Fu concentration added to cells was $10 \%$ of the $\mathrm{IC}_{50}$ (defined as the concentration producing a $50 \%$ reduction in absorbance at $540 \mathrm{~nm}$ compared with untreated controls in an MTT (3-[4, 5-dimethylthiazol2-yl]-2, 5-diphenyltetrazolium bromide) assay, and the concentration was increased by $10 \%$ every 2 weeks. $5-\mathrm{Fu}$ resistant single clones were picked up and pooled for the following experiments. All resistant cells were re-selected by the treatments every four weeks.

\section{Western blot analysis}

Cells were suspended in lysis buffer $(150 \mathrm{mM} \mathrm{NaCl}$, $100 \mathrm{mM}$ Tris, pH7.4, $5 \mathrm{mM}$ EDTA and $1 \%$ Triton $\mathrm{X}-100)$ containing a mixture of protease and phosphatase inhibitors. After centrifugation (10 $\mathrm{min}$ at $10,000 \mathrm{~g}$ ), the protein concentration was measured in triplicate in the supernatant by using the Bio-Rad Detergent Compatible protein assay (Bio-Rad, Richmond, CA) and protein aliquots were done. Equal amounts of protein $(75 \mu \mathrm{g})$ were separated by SDS-PAGE (12\%) and transferred onto nitrocellulose membrane. To verify the protein loading, membranes were stained with Ponceau red. Blots were blocked overnight at $4^{\circ} \mathrm{C}$ with $5 \%$ non-fat milk in TBS with $0.05 \%$ Tween 20 and probed with primary antibody. After incubation with the secondary antibody, immunological complexes were visualized by chemiluminescence detection according to the manufacturer's recommendations (Amersham Pharmacia Biotech, Arlington Heights, IL). The expression levels of Glut1 and $\beta$-actin in untreated and treated cells were determined by Chemiluminescence signals on films after Western blotting of 2 or 3 experiments.

\section{Cell viability assay}

A colorimetric assay using the tetrazolium salt, MTT, was used to assess the cytotoxicity of anticancer agents
5-Fu. Single-cell suspensions were prepared, and cell density was measured. MTT assays were performed according to the manufactory's instruction. Briefly, an equal number of cells were added into each well with culture medium containing normal concentration of drug or PBS (for untreated $100 \%$ survival control) was added. After 4 days culture, $0.1 \mathrm{mg}$ of MTT was added to each well and was incubated at $37^{\circ} \mathrm{C}$ for an additional $4 \mathrm{~h}$. Plates were centrifuged at $450 \mathrm{~g}$ for $5 \mathrm{~min}$ at room temperature, and the medium was removed. DMSO $(0.15 \mathrm{ml})$ was added to each well to solubilize the crystals, and the plates were immediately read at $540 \mathrm{~nm}$ on a scanning multiwell spectrometer (Bio-Tek instruments Inc., Burlington, VT). All of the experiments were performed three times.

\section{Real-time PCR}

RNA was extracted from cancer cells using the TRIzol reagent (Invitrogen, Carlsbad, CA, USA). The cDNA synthesis was performed using a SuperScript First-Standard Synthesis System for RT-PCR (Invitrogen) according to the manufacturer's protocol. Quantitative PCR analyses were performed using Assay-on-Demand primers and the TaqMan Universal PCR Master Mix reagent (Applied Biosystems, Foster City, NJ, USA). The samples were analysed using an ABI Prism 7700 Sequence Detection System (Applied Biosystems). The primers for q-PCR were: Glut1: Forward: 5'-AACTCTTCAGCCAGGGTCCAC-3'; Reverse: 5'-CACAGTGAAGATGATGAAGAC-3'. The expression levels of $\beta$-actin were used to normalize the relative expression levels. Experiments were triplicated.

\section{Plasmid DNA transfection}

Overexpression vectors containing wild type Glut1 and control vector were purchased from Origene. Transfection was performed using the Oligofectamine Transfection reagent (Invitrogen) according to the manufacturer's protocol. Forty-eight hours after transfection, cells were collected and prepared for further analysis by Western blot and chemo cytotoxicity assay.

\section{Glucose uptake assay}

Cells were seeded in 12 -well plates at $1 \times 10^{5}$ to $3 \times 10^{5}$ cells per well. Culture media was collected at 24 hours and stored at -20 degree until assayed. Glucose uptake was measured using an Amplex Red Glucose/Glucose Oxidase assay kit (Molecular Probes). Absorbance was measured at $563 \mathrm{~nm}$ using a SpectraMax M5 plate reader (Molecular Devices) and the results were normalized to the amount of total protein compared with the control cells.

\section{Colony Formation Assay}

For chondrosarcoma cell colony formation assay, 500 cells were seeded on $10 \mathrm{~cm}$ dish with drugs in regular cell culture medium. The medium was refreshed each two days with drugs. Cells were grown for three weeks and the surviving colonies were stained with gentian violet after methanol fixation, and visible colonies (>50 cells) were counted. Colonies from randomly-selected image areas of three replicate wells were enumerated in the present study protocol. 

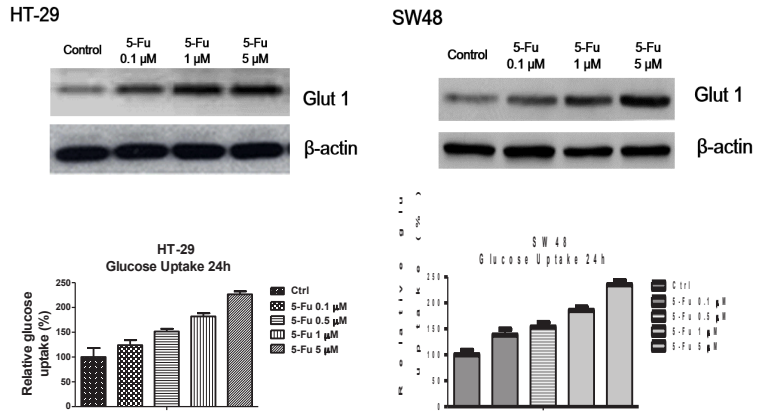

Figure 1. 5-Fu Treatments Induce Glut1 Expression in Colon Cancer Cells. (A) Western blotting experiments showed the expression of Glut1 were upregulated by $5-\mathrm{Fu}$ treatments at $0.1 \mu \mathrm{M}, 1 \mu \mathrm{M}$ and $5 \mu \mathrm{M}$ in HT-29 cells and SW48 cells. $\beta$-actin was used as a loading control. (B) Glucose uptake in two colon cells were measured under the 5 -Fu treatments at $0.1 \mu \mathrm{M}, 0.5 \mu \mathrm{M}, 1 \mu \mathrm{M}$ and $5 \mu \mathrm{M}$ for $24 \mathrm{hrs}$. The relative glucose uptake rates were calculated by the comparison to control treatment. Columns, mean of three independent experiments; bars, SE

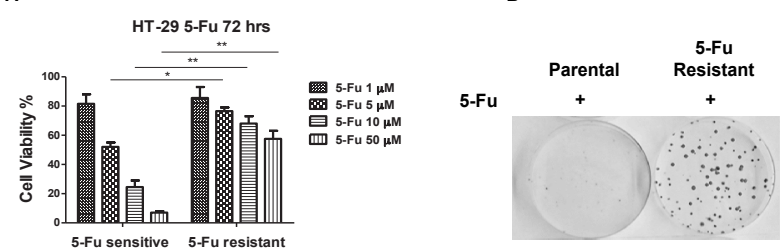

Figure 2. Generation of 5-Fu Resistant Cells from

HT-29. (A) HT-29 parental cells were treated with gradually increasing concentrations of $5-\mathrm{Fu}$ in regular cell culture conditions for selection of resistant cells as described in method. HT-29 5-Fu resistant clones were pooled and analyzed by the treatments of 5-Fu at indicated concentrations for $48 \mathrm{hrs}$ followed by the measurements of cell viability. All resistant cells were re-selected by the treatments every four weeks. (B) Clonogenic assay of HT-29 parental cells (5-Fu sensitive) and 5-Fu resistant cells were treated with 5-Fu for two weeks at $10 \mu \mathrm{M}$ and allowed to form colonies. Photographs of $10-\mathrm{cm}$ dishes in a representative experiment are shown. Columns, mean of three independent experiments; bars, SE. $* p<0.05 ; * * p<0.01$

\section{Statistical analysis}

The unpaired Student's t-test was used for the data analysis. All data were shown as mean \pm standard error (SE). A statistical difference of $p<0.05$ was considered significant.

\section{Results}

5-Fu treatment induces the expression of Glutl and promotes glucose uptake

Since Glut 1 is an important key enzyme of glycolysis, we started to examine the role of Glut 1 in mediating drug resistance in human colon cancer cells. The expression of Glut1 was examined in HT-29 and SW48 cell lines under treatments with low toxic 5-Fu for 24 hours. Interestingly, we found that Glut 1 levels were markedly induced under 5-Fu treatments in both human colon cancer cells (Figure 1A). Meanwhile, treatment with 5-Fu resulted in the induction of Glut1 expression in a dose-dependent pattern
A

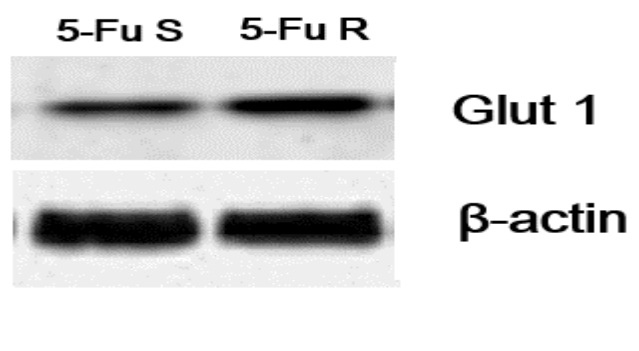

B

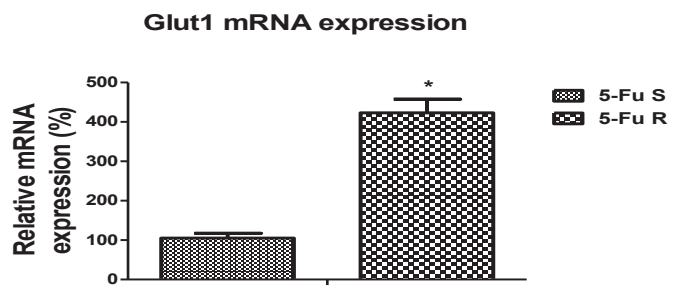

C
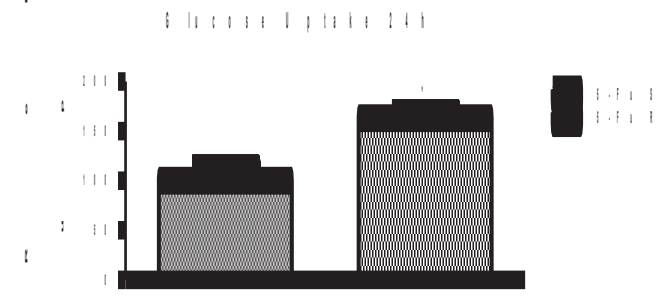

Figure 3. The Expression of Glut1 is Upregulated in 5-Fu Resistant Cells. (A) Western blotting experiments showed the protein level of GLUT1 was upregulated in 5-Fu resistant colon cancer cells. $\beta$-actin was used as a loading control. (B) mRNA levels of GLUT1 was increased in 5-Fu resistant cells measured by Real-time PCR. (C) Glucose uptake in 5-Fu resistant colon cells were measured after $24 \mathrm{hrs}$. The relative glucose uptake rates were calculated by the comparison to control treatment. Columns, mean of three independent experiments; bars, SE. ${ }^{*} p<0.05$

(Figure 1A). We further examined the glucose uptake rate of colon cancer cells in response to 5-Fu treatments. Consistently, the glucose uptake of HT-29 and SW48 cells were significantly increased by $5-\mathrm{Fu}$ treatments (Figure 1B). Taken together, our results suggested that 5-Fu resistance is correlated with the increased Glut1 expression.

Generation and characterization of 5-Fu resistant colon cancer cells

To investigate whether upregulated Glut 1 expression is the major mechanism for 5-Fu resistance, we selected 5-Fu resistant cells from human colon cancer cell line. HT-29 cells were treated with gradually increasing concentrations of 5-Fu in cell culture medium for selection of 5-Fu resistant cells. After successive treatments for duration of 3 months, several resistant cell clones were developed from the HT-29 cell line. The resistant clones were pooled and were used for all subsequent experiments in this study. To compare the survival capacity of both 5 -Fu sensitive and resistant cells, cells were treated with $5-\mathrm{Fu}$ at the indicated concentrations. Cell viability assay detected a 
A

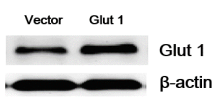

B
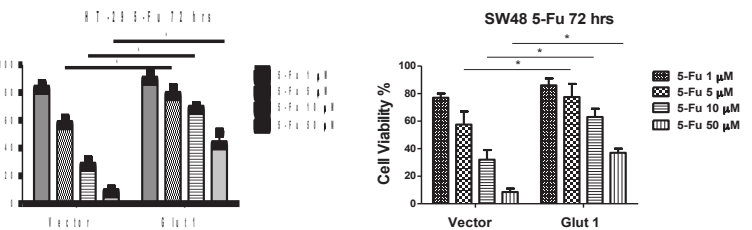

Figure 4. Overexpression of Glut1 Renders Colon

Cells Resistant to 5-Fu. Transient transfection of Glut1 into (A) HT-229 cells and (B) SW48 cells. Forty-eight hours later, vector control cells and cells with overexpression of Glut 1 were treated with 5-Fu with indicated concentrations for 72 hrs, then the cell viability assays were performed. $\beta$-actin was used as a loading control. Columns, mean of three independent experiments; bars, SE. ${ }^{*} p<0.05$
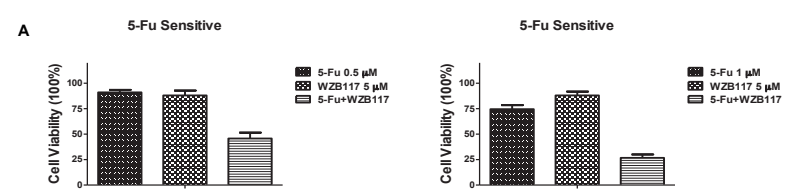

B

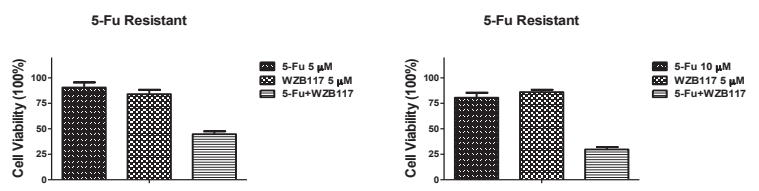

Figure 5. Inhibition of Glut1 by WZB117 Sensitizes HT-29 Cells to 5-Fu. (A) HT-29 5-Fu sensitive cells were treated with 5-Fu alone, WEB117 alone, and 5-Fu plus Web117 for $72 \mathrm{hrs}$ at the indicated concentrations followed by the measurements of cell viability. (B) HT-29 5-Fu resistant cells were treated with 5-Fu alone, WZb117 alone and 5-Fu plus WZB117 at the indicated concentrations for $72 \mathrm{hrs}$ followed by the measurements of cell viability. Columns, mean of three independent experiments; bars, SE

smaller percentage of dead cells in 5-Fu resistant cells at $10 \mu \mathrm{M}$ and $50 \mu \mathrm{M}$, compared to their parental $5-\mathrm{Fu}$ sensitive cells after treatment with $10 \mu \mathrm{M}$ and $50 \mu \mathrm{M}$ 5-Fu for $72 \mathrm{hrs}$ (Figure 2A). To support our results, we performed colony formation assay for the detection of clonogenicity of 5-Fu sensitive and resistant cells under treatments of $5-\mathrm{Fu}$. Consistent with the cell viability assay, results from Figure $2 \mathrm{~B}$ showed that $5-\mathrm{Fu}$ sensitive cells exhibited significant colony formation inhibition in fourteen days under the treatments of the 5-Fu sensitive cells compared with resistant cells. Taken together, our results showed that $5-\mathrm{Fu}$ resistant cells could tolerate much higher concentrations of 5-Fu treatment compared to sensitive cells.

Increased expression of Glut1 in 5-Fu resistant colon cancer cells

To examine the role of Glut1 in mediating $5-\mathrm{Fu}$ resistance in colon cancer cells, the expression of Glut1 was examined in $5-\mathrm{Fu}$ sensitive and resistant cells respectively. We found that Glut1 levels were increased in 5-Fu resistant cells, compared to their parental cells (Figure 3A). The mRNA of Glut1 was also increased about 4-fold in resistant cells, compared to parental cells (Figure 3B). Consistently, the 5-Fu resistant cells showed a higher glucose uptake rate (Figure 3C). To further strengthen our above results, we transiently transfected Glut1 to exogenously overexpress glycolic key enzyme in colon cancer cells. As we expected, HT-29 and SW48 cells with overexpression of Glut1 obtained resistance to 5-Fu, cells with higher expression of Glut1 exhibited more survival abilities under multiple concentrations of 5-Fu (Figure 4A\&4B), indicating dysregulated glucose metabolism contributes to 5-Fu resistance in colon cancer cells. These results revealed the expression of Glut 1 and glucose metabolism were involved in chemoresistance, suggesting Glut1 might be a putative therapeutic target for overcoming 5-Fu resistance in colon cancer.

\section{Inhibition of Glut1 re-sensitizes 5-Fu resistant cells to} $5-F u$

The upregulated Glut1 expression detected in 5-Fu resistant cells suggests that Glut1 may play a critical role in 5-Fu resistance. We hypothesized that the downregulation of Glut 1 by specific inhibitor might sensitize 5-Fu resistant cells to 5-Fu. Therefore, the effect of Glut1 inhibition on the sensitivity of 5-Fu treatment was investigated. It has been reported a small molecule inhibitor of Glut1WZB117 can efficiently inhibit the expression of Glut1, down-regulate glycolysis and inhibits cancer cell growth (Wang et al., 1993). To address this point, HT-29 5-Fu sensitive and resistant cells were treated with 5-Fu alone, WZB117 alone and the combination of 5-Fu and WZB117 at indicated concentrations to investigate the effects of the combination of 5-Fu with Glut1 inhibitor on 5-Fu resistant colon cancer cells. The treatment with either 5-Fu or WZB117 alone at low concentrates did not induce significant cell death. However, in both 5-Fu sensitive (Figure 5A) and resistant (Figure 5B) HT-29 cells, 5-Fu combined with WZB117 were much more effective in inhibiting cell viability compared with either agent given alone, indicating the combination of 5-Fu and Glut1 inhibitor displayed a synergistic inhibitory effect on colon cancer cells compared to either agent given alone.

\section{Discussion}

In this report, we have identified cells with induced glucose uptake under low toxic 5-Fu treatment and analyzed the proteins implicated in glucose metabolic pathway that might be used as markers to predict defect of tumor responsiveness to 5-Fu therapy. To do this, we have generated, for several weeks, a 5-FU resistant colon cancer cell line that displayed upregulated Glut1 expression compared with 5-Fu sensitive cells.

5 -Fu is used frequently in chemotherapy for a variety of solid tumors. However, development of drug resistance in human tumor cells is the primary cause of failure of chemotherapy. Multiple mechanisms responsible for the drug resistant phenotype in cancer cells have been recognized. Various mechanisms have been proposed to 
explain resistance to 5-FU. The most common mechanism is characterized by the correlation with either defective drug uptake or alterations in the activities of anabolic and/or catabolic enzymes (Beck et al., 1994; Wang et al., 1993). In addition, resistance to 5-Fu have been attributed to thymidylate synthase (TS), which is a crucial enzyme in de novo synthesis of thymidylate (Curtin et al., 1991). Our study provides a novel mechanism for 5 -Fu resistance in colon cancer cells. We observed the Glut1 expression was upregulated in 5-Fu resistant cancer cells and exogenous overexpression of Glut1 facilitated colon cancer cells obtain resistance to $5-\mathrm{Fu}$. Therefore, the upregulated glucose uptake and Glut1 expression can be the potential target for the development of anticancer agent.

The 'Warburg effect' described that in contrast to their normal counterparts, cancer cells prefer to utilize aerobic glycolysis for energy generation rather than mitochondrial oxidative phosphorylation (Vander et al., 2009). Thus, the metabolic dependencies of cancer cells can be exploited for cancer treatment by the inhibition of metabolic enzymes to improve the efficacy of cancer therapy. Inhibition of glucose uptake has been studied to sensitize cancer cells to daunorubicin (Vander et al., 2009; Zhao et al., 2013). A recent study reported inhibition of dehydrogenase kinase-1 (PDK-1) by specific inhibitor DCA sensitized gastric cancer cells to 5-Fu, indicating suppression of glucose metabolism in cancer cells might be an effectively therapeutic method for the overcoming of anticancer drug resistance (Hur et al., 2013). Another study demonstrated targeting dysregulated glucose metabolism in breast cancer cells led to overcome Trastuzumab resistance (Zhao et al., 2011). In addition, several other glycolytic inhibitors with promising anticancer activity are currently at various stages of preclinical and clinical development. It has been reported that WZB117-treated cancer cells undergo senescence and necrosis, rather than apoptosis, through the depletion of intracellular ATP so much that the cancer cells were unable to carry out apoptosis, forcing the cells to undergo senescence and necrosis (Liu et al., 2012). Our study is the first time that a synergistic inhibitory effect is observed in colon cancer cells treated by the combination of 5-Fu and Glut1 inhibitor. However, the detailed mechanism for the anticancer effect by the combination of Glut1 inhibitor and 5-Fu is still unclear. Our next project will focus on the gene expression profiling of colon cancer cells under this combined treatment. Moreover, we will investigate other Gluts inhibitors such as Fasentin (Wood et al., 2008) and Phloretin (Wu et al., 2009) which were also shown to be effective in reducing cancer cell growth to explore more effective compounds to be utilized as potential anticancer agents for the development of therapeutic strategies.

\section{Acknowledgements}

No potential conflicts of interest were disclosed.

\section{References}

Beck A, Etienne MC, Cheradame S, et al (1994). A role for dihydropyrimidine dehydrogenase and thymidylate synthase in tumour sensitivity to fluorouracil. Eur J Cancer, 30, 1517-22.

Boyer J, McLean EG, Aroori S, et al (2004). Characterization of p53 wild-type and null isogenic colorectal cancer cell lines resistant to 5-fluorouracil, oxaliplatin, and irinotecan. Clin Cancer Res, 10, 2158-67.

CarvalhoKC,Cunha IW, Rocha RM, etal(2011).GLUT1 expression in malignant tumors and its use as an immunodiagnostic marker. Clinics, 66, 965-72.

Curtin NJ, Harris AL, Aherne GW (1991). Mechanism of cell death following thymidylate synthase inhibition: 2'-deoxyuridine5 '-triphosphate accumulation, DNA damage, and growth inhibition following exposure to CB3717 and dipyridamole. Cancer Res, 51, 2346-52.

DeBraud F, Munzone E, Nole F, et al (1998). Synergistic activity of oxaliplatin and 5-fluorouracil in patients with metastatic colorectal cancer with progressive disease while on or after 5-fluorouracil. Am J Clin Oncol, 21, 279-83.

Herrmann R (1996). 5-Fluorouracil in colorectal cancer, a never ending story. Ann Oncol, 7, 551-2.

Hur H, Xuan Y, Kim YB, et al (2013). Expression of pyruvate dehydrogenase kinase-1 in gastric cancer as a potential therapeutic target. Int J Oncol, 42, 44-54.

Li LJ, Li GD, Wei HL, et al (2014). Insulin resistance reduces sensitivity to Cis-platinum and promotes adhesion, migration and invasion in HepG2 cells. Asian Pac J Cancer Prev, 15, 3123-8.

Liu Y, Cao Y, Zhang W, et al (2012). A small-molecule inhibitor of glucose transporter 1 downregulates glycolysis, induces cell-cycle arrest, and inhibits cancer cell growth in vitro and in vivo. Mol Cancer Ther, 11, 1672-82.

Longley DB, Harkin DP, Johnston PG (2003) 5-fluorouracil: mechanisms of action and clinical strategies. Nat Rev Cancer, 3, 330-8.

Peters GJ, van Triest B, Backus HH, et al (2000). Molecular downstream events and induction of thymidylate synthase in mutant and wild-type p53 colon cancer cell lines after treatment with 5-fluorouracil and the thymidylate synthase inhibitor raltitrexed. Eur J Cancer, 36, 916-24.

Subbarayan PR, Lee K, Ardalan B (2010). Arsenic trioxide suppresses thymidylate synthase in 5-FU-resistant colorectal cancer cell line HT29 In Vitro re-sensitizing cells to 5-FU. Anticancer Res, 30, 1157-62.

Vander HM, Cantley LC, Thompson CB (2009). Understanding the Warburg effect: the metabolic requirements of cell proliferation. Science, 324, 1029-33.

Wang FS, Aschele C, Sobrero A, et al (1993). Decreased folylpolyglutamate synthetase expression: a novel mechanism of fluorouracil resistance. Cancer Res, 53, 3677-80.

Wang YD, Li SJ, Liao JX (2013). Inhibition of glucose transporter 1 (GLUT1) chemosensitized head and neck cancer cells to cisplatin. Technol Cancer Res Treat, 12, 525-35.

Wood TE, Dalili S, Simpson CD, et al (2008). A novel inhibitor of glucose uptake sensitizes cells to FAS-induced cell death. Mol Cancer Ther, 7, 3546-55.

Wu CH, Ho YS, Tsai CY, et al (2009). In vitro and in vivo study of phloretin-induced apoptosis in human liver cancer cells involving inhibition of type II glucose transporter. Int $J$ Cancer, 124, 2210-9.

Zhang N, Yin Y, Xu SJ, et al (2008). 5-Fluorouracil: mechanisms of resistance and reversal strategies. Molecules, 13, 1551-69.

Zhao Y, Butler EB, Tan M (2013). Targeting cellular metabolism to improve cancer therapeutics. Cell Death Dis, 4, 532.

Zhao Y, Liu H, Liu Z, et al (2011). Overcoming trastuzumab resistance in breast cancer by targeting dysregulated glucose metabolism. Cancer Res, 71, 4585-97.

Zhao L, Wang WJ, Zhang JN, et al (2014). 5-Fluorouracil and Interleukin-2 immunochemotherapy enhances immunogenicity of Non-Small cell lung cancer A549 cells through upregulation of NKG2D ligands. Asian Pac J Cancer Prev, 15, 4039-44. 